Erklärung der Abbildungen auf Taf. I. und II.

\title{
Tafel I.
}

Fig. 1. Larve von Rhodites Rosae nahe der Verpuppung mit ihrem Tracheensystem, von oben gesehen.

- 2. Reife Larve von Aulax Hieracii mit ihrem Tracheensystem, von der Seite.

- 3. Larve von Aulax Hieracii kurz vor der Verpuppung mit ihrem Tracheensystem, von der Seite.

- 4. Hinterende des Haupttracheenstammes der Larve von $\boldsymbol{A u}$ lax Hieracii mit den sich ablösenden Stigmenästen.

- 5. Puppe von Aulax Hieracii $\sigma^{\nearrow}$. Das Stigma am vorletzten Segmente zeigt noch keine hakenförmigen Schläuche.

- 6. Hinterleib der Puppe von Aulax Hieracii 9. Am Stigma des vorletzten Segments sind die hakenförmigen Schläuche sowie in den vorhergehenden Segmenten die Narben der früheren Stigmen sichtbar.

- 7. Hälfte des siebenten Hinterleibssegments von Aulax Hieracii mit dem Stigma, dem hinteren Ende des Haupttracheenstammes und einem Stück des hintern Querstranges. Am Stigma die Reste der hakenförmigen Schläuche.

- 8. Mandibel der Larve von Aulax Hieracii.

- 9. - - - - Decatoma.

- $10 . \quad-\quad-\quad$ - Pteromalus.

-11. - - - Eupelmus.

-12. - - Callimome.

\section{Tafel II.}

- 13. Puppe von Rhopalotus cothurnatus 9 von der Rückenseite. st. st. Metathorax- und Hinterleibsstigma.

- 14. Dieselbe von der Bauchseite; am Hinterrande des sechsten Bauchsegments das Grübchen der künftigen Geschlechtsöfnung. - st. Hinterleibsstigma.

- 15. Hintere Hälfte der Puppe von Rhopalolus cothurnalus ơ mit dem dem Penis entsprechenden Höcker des achten Bauchsegments. 
Fig. 16. Larve von Formica mit ihren Stigmen.

- 17. Larve von Apis mellifica mit den Stigmen.

- 18. Thorax von Vespa crabro, von der Seite. A. derselbe ganz, $\boldsymbol{B}$. derselbe in seine Bestandtheile zerlegt.

1. Pronotum, an der bewimperten Stelle des Hinterrandes die Lage des Stigma andeutend.

2. Prosternum.

3. Vorderhüfte.

4. Mesoscutum mit dem Flügelschüppchen.

5. Scutellum.

Fig. 19. Thorax von Tenthredo atra. A. ganz, B. in seine Bestand theile zerlegt.

1. Pronotum.

2. Prosternum.

3. Episternen des Prothorax.

4. Vorderhüfte.

5. Mesoscutum mit d. Scutellum.

6. Mesophragma.

7. Mesoslernum.
6. Mesophragma.

8. Mittelhüfte.

9. Metanotum.

10. Metasternum mit dem Dorsalhalbringe des 4. Segments verschmolzen.

11. Hinterhüfte.
7. Mesosternum.

Fig. 20. Kopf und Thorax einer Ctenophora (Tipulid.)

\section{Prothorax.}

2. Vorderhïfte.

3. Mesonotum.

4. Mesophragma.

5. Mesosternum. 5'. Epimerum.
8. Episternum/ des

9. Epimerum Mesothorax.

10. Mittelhüfte.

11. Mietanotum.

12. Metasternum.

13. Hinterhüfte.

14. Dorsalhalbring d.4.Segmenls.

6. Millelhüfte.

7. Metanotum.

8. Metasternum mit dem Epimerum.

9. Hinterhüfte.

Fig. 21. A. Thorax einer Ctenophora) in der Mittellinie senkrecht $\boldsymbol{B}$. Thorax einer Hornisse durchschn. (halbschematisch).

a. grofser Rückenmuskel, abaisseur des ailes.

b. Schildchen.

c. Metanotum. 


\section{$2 \mathrm{BHL}$ Biodiversity Heritage Library}

1865. "Erklärung der Abbildungen auf Taf. I. und II." Berliner entomologische Zeitschrift / herausgegeben von dem Entomologischen Vereine in Berlin 9(3ロ4), 217-218. https://doi.org/10.1002/mmnd.18650090305.

View This Item Online: https://www.biodiversitylibrary.org/item/34938

DOI: https://doi.org/10.1002/mmnd.18650090305

Permalink: https://www.biodiversitylibrary.org/partpdf/210235

\section{Holding Institution}

Smithsonian Libraries

\section{Sponsored by}

Smithsonian

\section{Copyright \& Reuse}

Copyright Status: Public domain. The BHL considers that this work is no longer under copyright protection.

This document was created from content at the Biodiversity Heritage Library, the world's largest open access digital library for biodiversity literature and archives. Visit BHL at https://www.biodiversitylibrary.org. 